Proceedings of the 2nd International Congress APMAS2012, April 26-29, 2012, Antalya, Turkey

\title{
Fast Speed Semiconductor Ring Lasers Using Optical Injection Locking
}

\author{
M.I. $\operatorname{MEMON}^{a, b}$, H. FATHALlah ${ }^{a}$ AND S. YU ${ }^{c}$ \\ ${ }^{a}$ KACST Technology Innovation Center RFTONICS, King Saud University, Riyadh, Saudi Arabia \\ ${ }^{b}$ COMSATS Institute of Information Technology, Islamabad, Pakistan \\ ${ }^{c}$ University of Bristol, Bristol, United Kingdom
}

\begin{abstract}
Theoretical study for frequency response and modulation bandwidth of slave semiconductor ring laser in the master-slave configuration using optical injection locking has been investigated. Equations for frequency response of optical injection locking-semiconductor ring laser to the direct modulation, amplitude modulation of master laser, respectively, are derived and simulated. Enhancement in the modulation bandwidth of $>100 \mathrm{GHz}$ is reported between negative to positive detuning frequency and increasing injection power ratio.
\end{abstract}

DOI: 10.12693/APhysPolA.123.180

PACS: 42.55.Px, 42.55.Sa, 42.60.By, 42.60.Fc, 42.65.Pc

\section{Introduction}

In the modern optical communication networks, one of the most important figures of merit that decides the achievable data rate is the modulation bandwidth of the semiconductor laser (SL) source. In SL optical injection locking (OIL) has been found useful in achieving huge modulation bandwidths. Previously, several works have been proposed on the enhancement of slave laser's bandwidth in the master-slave configuration due to OIL [1]. Though, as the backward light from the slave laser is stopped by isolator, these systems are not suitable for coplanar integration.

Semiconductor ring laser (SRL) operates in the direction of external optical injection thus it eliminates the need of isolator [2]. Previously the frequency response and the modulation bandwidth of SRL are investigated in detail experimentally [3]. In this paper theoretical study of the frequency response of OIL-SRL is proposed. It has been proved using different modulation schemes (direct, amplitude and phase modulation) that OIL SRL as a slave in the master-slave configuration shows huge enhancement in the bandwidth $>100 \mathrm{GHz}$.

\section{Theory}

SRL works in the direction of injection and modulated light is injected in the counterclockwise (CCW) direction into the SRL. Without loss of generality, the rate equation of OIL-SRL is only considered in CCW direction and can be expressed as

$$
\begin{aligned}
& \frac{\mathrm{d} S(t)}{\mathrm{d} t}=\Gamma v_{\mathrm{g}} g_{\mathrm{n}}\left[N(t)-N_{\mathrm{tr}}\right]\left[1-\varepsilon_{s} S(t)\right] S(t)-\frac{S(t)}{\tau_{p}} \\
& \quad+2 \kappa_{\mathrm{inj}} \sqrt{S(t) S_{\mathrm{inj}}(t)} \cos \left(\varphi(t)-\varphi_{\mathrm{inj}}(t)\right), \\
& \frac{\mathrm{d} \varphi(t)}{\mathrm{d} t}=\frac{\alpha}{2} \Gamma v_{\mathrm{g}} g_{\mathrm{n}}\left[N(t)-N_{\mathrm{tr}}\right]\left[1-\varepsilon_{s} S(t)\right]-\frac{\alpha}{2 \tau_{p}}
\end{aligned}
$$

$$
\begin{aligned}
& -\kappa_{\mathrm{inj}} \sqrt{\frac{S_{\mathrm{inj}}(t)}{S(t)}} \sin \left(\varphi(t)-\varphi_{\mathrm{inj}}(t)\right)-\Delta \omega_{\mathrm{inj}}, \\
& \frac{\mathrm{d} N(t)}{\mathrm{d} t}=\frac{\eta_{i} I(t)}{q V}-\frac{N(t)}{\tau_{N}} \\
& -v_{\mathrm{g}} g_{\mathrm{n}}\left[N(t)-N_{\mathrm{tr}}\right]\left[1-\varepsilon_{s} S(t)\right] S(t),
\end{aligned}
$$

where $S(t)$ and $\varphi(t)$ are the output photon density and the phase in the CCW direction, respectively. The parameters $\alpha, \Gamma, v_{\mathrm{g}}, g_{\mathrm{n}}, N, N_{\mathrm{tr}}, \varepsilon_{s}, \tau_{p}, \eta_{i}, I, \mathrm{q}$, and $\tau_{N}$ are line-width enhancement factor, optical confinement factor, group velocity, differential gain at transparency, carrier density, carrier density at transparency, self-gain saturation coefficient, photon life-time, the quantum efficiency of the bias current $I$, the electron charge in the volume of active region $V$ and carrier lifetime, respectively. $\Delta \omega_{\text {inj }}$ is the detuning frequency and it can be defined as $\Delta \omega_{\text {inj }}=\omega_{M}-\omega_{f}$, where $\omega_{M}$ and $\omega_{f}$ are lasing frequencies of the master laser and SRL respectively. $\kappa_{\text {inj }}$ represents the field coupling coefficient of the optical injection into the SRL cavity and it can be defined as $\kappa_{\text {inj }}=\sqrt{T /(1-T)} / \tau_{l}$, where $T$ and $\tau_{l}$ are the output coupling ratio and round-trip time in the laser cavity, respectively.

\section{Steady state solution}

This product $\varepsilon_{s} S \ll 1$ and has no significant effect. Thus for the steady-state solution and small signal analysis, the terms involving $\varepsilon_{s} S$ may be neglected. Free-running steady state solution can be obtained by setting the derivatives and the injection terms to zero in Eqs. (4)-(6) thus the analytic solution for photon density can be given as

$$
S_{f}=\tau_{p} \Gamma\left(\frac{\eta_{i} I}{q V}-\frac{N_{f}}{\tau_{N}}\right), \quad N_{f}=N_{\mathrm{tr}}+\frac{1}{\tau_{p} \Gamma v_{\mathrm{g}} g_{\mathrm{n}}} .
$$

For the external optical injection from the master laser, $\varphi_{\text {injo }}$ is assumed to be zero so the dc SRL phase $\varphi_{0}$ represents the total dc phase of the master and slave lasers. 
Using the above results and solving Eqs. (4)-(6) with external optical injection, the steady state solution can be found in the similar fashion as

$$
\begin{aligned}
& S_{0}=\frac{S_{f}-\frac{\tau_{p} \Gamma \Delta N}{\tau_{N}}}{1+\tau_{p} \Gamma v_{\mathrm{g}} g_{\mathrm{n}} \Delta N}, \\
& \varphi_{0}=\arcsin \left(-\frac{\Delta \omega_{\mathrm{inj}}}{\kappa_{\mathrm{inj}} \sqrt{\frac{S_{\mathrm{inj} 0}}{S_{0}}\left(1+\alpha^{2}\right)}}\right)-\arctan \alpha, \\
& \Delta N=-\frac{2 \kappa_{\mathrm{inj}}}{\Gamma v_{\mathrm{g}} g_{\mathrm{n}}} \sqrt{\frac{S_{\mathrm{inj} 0}}{S_{0}}} \cos \varphi_{0},
\end{aligned}
$$

where $S_{0}, \varphi_{0}$ and $\Delta N$ are photon density, phase, and carrier density difference, respectively, at steady state of injection locked SRL. Steady state value of carrier density is $N_{0}=\Delta N+N_{f}$ and $\sqrt{S_{\text {injo }} / S_{f}}$ is the injection ratio.

Detuning frequency can be determined using above equations as

$$
\Delta \omega_{\text {inj }}=-\kappa_{\text {inj }} \sqrt{R_{\text {inj }}\left(1+\alpha^{2}\right)} \sin \left(\varphi_{0}+\arctan \alpha\right)
$$

\section{Small signal analysis}

By linearising Eqs. (4)-(6) the differential equation system may be placed in the matrix form as

$$
\left[\begin{array}{c}
\delta S \\
\delta \varphi \\
\delta N
\end{array}\right]=\boldsymbol{M}^{-1} \boldsymbol{X}
$$

where

$$
\boldsymbol{M}=\left[\begin{array}{ccc}
a_{11}+\mathrm{j} \omega & a_{12} & a_{13} \\
a_{21} & a_{22}+\mathrm{j} \omega & a_{23} \\
a_{31} & a_{32} & a_{33}+\mathrm{j} \omega
\end{array}\right] .
$$

The elements of state transition matrix $M$ can be given as $a_{11}=z \cos \varphi_{0}, a_{12}=2 z S_{0} \sin \varphi_{0}, a_{13}=$ $-\Gamma v_{\mathrm{g}} g_{\mathrm{n}} S_{0}, a_{21}=-z \sin \varphi_{0} / 2 S_{0}, a_{22}=z \cos \varphi_{0}, a_{23}=$ $-\alpha \Gamma v_{\mathrm{g}} g_{\mathrm{n}} / 2, a_{31}=\left(1 / \tau_{p}-2 z \cos \varphi_{0}\right) / \Gamma, a_{32}=0$ and $a_{33}=1 / \tau_{N d}+v_{\mathrm{g}} g_{\mathrm{n}} S_{0}$, where $z=\kappa_{\mathrm{inj}} \sqrt{S_{\mathrm{inj}} / S_{0}}$, and $\tau_{N d}$ is differential carrier life time and it can be given as

$$
\tau_{N d}=1 / A_{\mathrm{g}}+2 B_{\mathrm{g}} N_{0}+3 C_{\mathrm{g}} N_{0}^{2},
$$

where $A_{\mathrm{g}}, B_{\mathrm{g}}$ and $C_{\mathrm{g}}$ are nonradiative coefficient, radiative coefficient and Auger recombination coefficient, respectively. $X$ is the input vector and driven separately in accordance with the modulation scheme. In the case of direct modulation of OIL-SRL, the continuous wave light is injected from the master laser to the slave SRL. Classically for direct modulation response the output is photon density modulation and thus the frequency response can be given as

$$
H_{\mathrm{dir}}(\mathrm{j} \omega)=\frac{\delta S}{\delta I}=-\frac{\eta_{i}}{q V} \frac{a_{13}\left(\mathrm{j} \omega+Z_{d}\right)}{\operatorname{det}(\boldsymbol{M})},
$$

where $Z_{d}$ is the zero of the system and it may expressed as $Z_{d}=\left(a_{12} a_{23}-a_{22} a_{13}\right) / a_{13}$ and $\operatorname{det}(\boldsymbol{M})$ is the determinant of state-transition matrix $\boldsymbol{M}$.

For amplitude modulation, the output is the photon density modulation of the slave SRL and the input is the amplitude modulated signal from the amplitude modulator. Thus the frequency response for amplitude modulated OIL-SRL system may be given as

$$
\begin{aligned}
& H_{A M}(\mathrm{j} \omega)=\frac{\delta S}{\delta S_{\mathrm{inj}}} \\
& =M_{A M} \frac{\left(\mathrm{j} \omega+Z_{A M 1}\right)\left(\mathrm{j} \omega+Z_{A M 2}\right)}{\operatorname{det}(\boldsymbol{M})},
\end{aligned}
$$

where $M_{A M}=z\left(S_{0} / S_{\text {injo }}\right) \cos \varphi_{0}$ and $Z_{A M 1}$ and $Z_{A M 2}$ are zeros and they may be expressed as: $Z_{A M 1}=a_{33}=\left(1 / \tau_{N d}\right)+v_{\mathrm{g}} g_{\mathrm{n}} S_{0}, Z_{A M 2}=z / \cos \varphi_{0}$ Similarly for phase modulated injection locking system, the input is phase modulated light signal injected from the phase modulator and the output is the phase modulation of the slave laser and it can be given as

$$
H_{P M}(\mathrm{j} \omega)=\frac{\delta \varphi}{\delta \varphi_{\mathrm{inj}}}=\frac{-a \omega^{2}+b \omega+C}{\operatorname{det}(\boldsymbol{M})},
$$

where

$$
a=z \cos \varphi_{0}, \quad b=z^{2}\left(1+a_{33} \cos ^{2} \varphi_{0}\right) .
$$

\section{Simulation results and discussion}

Equations (4)-(6) are simulated using the laser parameters given in Table and they are of the SRL used in [3]. The parameters $n, L, W_{\mathrm{wg}}$ and $T_{\mathrm{qw}}$ are group refractive index, length of the SRL cavity, width of the waveguide, and thickness of the quantum well, respectively. All the other parameters are defined in the above section.

Injection locked SRL parameters.

TABLE

\begin{tabular}{c|c|c|c}
\hline \hline Symbol & Value & Symbol & Value \\
\hline$n$ & 3.41 & $N_{\mathrm{qw}}$ & 5 \\
$\alpha$ & 2.52 & $g_{\mathrm{n}}$ & $6 \times 10^{-20} \mathrm{~m}^{2}$ \\
$L$ & $1406 \mu \mathrm{m}$ & $\Gamma$ & 0.62 \\
$W_{\mathrm{wg}}$ & $2 \mu \mathrm{m}$ & $\eta_{i}$ & 0.5 \\
$T_{\mathrm{qw}}$ & $6 \mathrm{~nm}$ & $N_{\mathrm{tr}}$ & $1.25 \times 10^{24} \mathrm{~m}^{-3}$ \\
$T$ & 0.5 & $A_{\mathrm{g}}$ & $2.1 \times 10^{8} \mathrm{~s}^{-1}$ \\
$B_{\mathrm{g}}$ & $4.5 \times 10^{-10} \mathrm{~cm}^{3} / \mathrm{s}$ & $C_{\mathrm{g}}$ & $5.83 \times 10^{-29} \mathrm{~cm}^{6} / \mathrm{s}$
\end{tabular}

The first important issue is the stable locking range and it is simulated between detuning frequency and optical injection ratio as shown in Fig. 1. Detuning frequency is the frequency difference between master laser and the slave SRL.

The frequency response broadens with the increase in the injection ratio as shown in Fig. 2 using direct, amplitude modulation and phase modulation, respectively. It is shown in Fig. 2a that this low-frequency roll-off factor shrinks the $3 \mathrm{~dB}$ frequency with increasing injection ratio. The benefit of using intensity modulation is that dependence on the roll-off factor is decreased with the increase in the injection ratio. In Fig. 2c response is simulated as linear function of ratio of output phase of the 


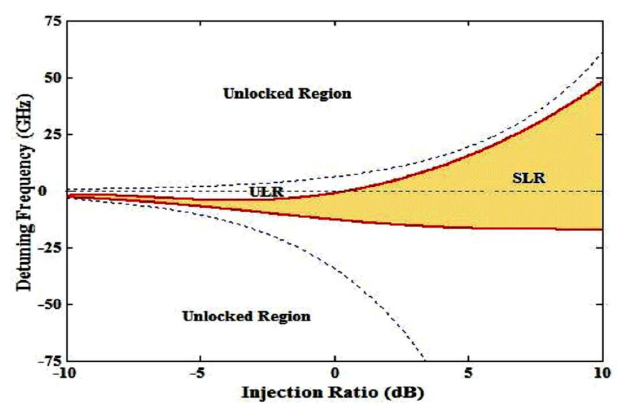

Fig. 1. Locking range of SRL illustrates stable locking range (SLR) and unstable locking range (ULR).

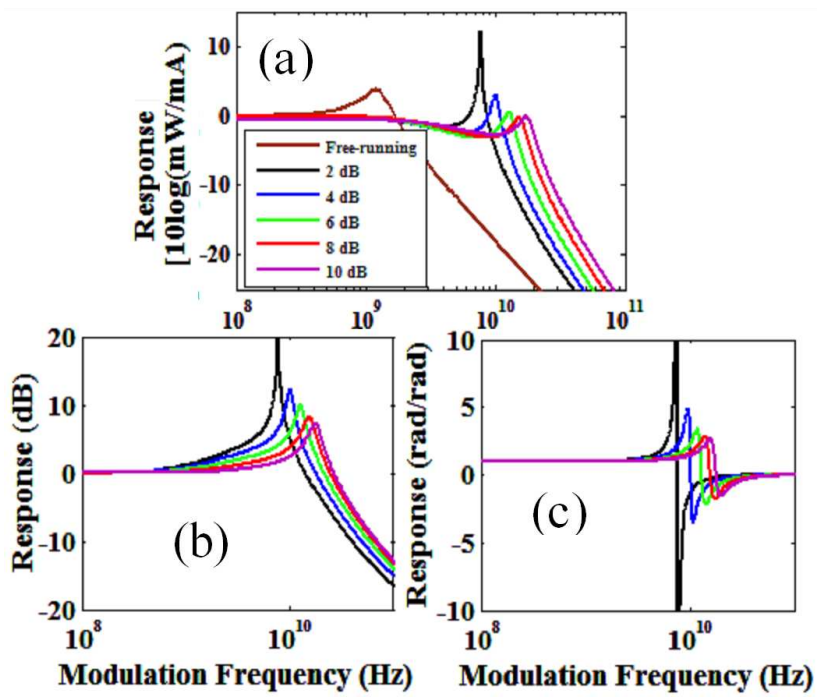

Fig. 2. Frequency response of OIL SRL using (a) direct modulation, (b) amplitude modulation, and (c) phase modulation with changing optical injection.

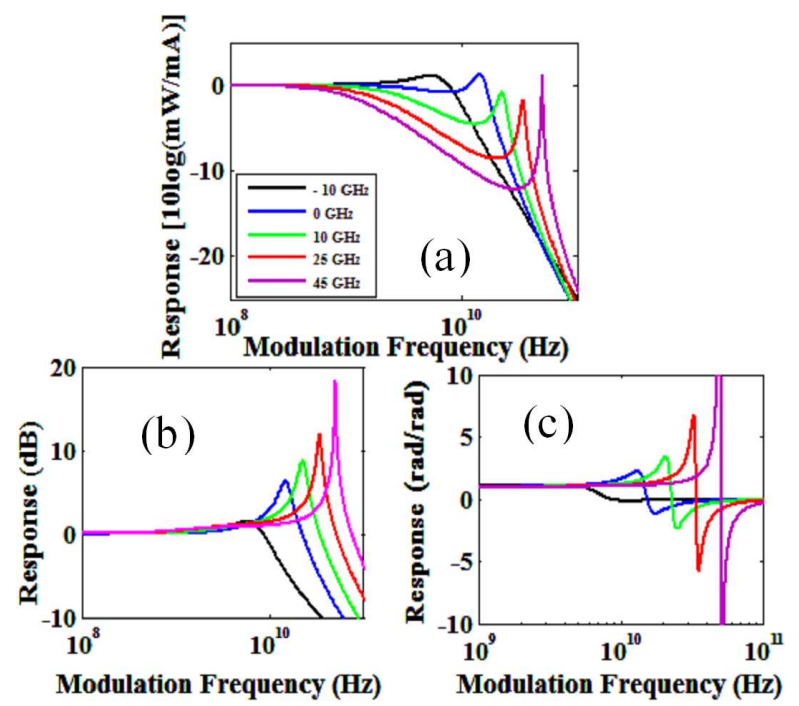

Fig. 3. Frequency response of OIL-SRL using (a) direct modulation, (b) amplitude modulation, (c) phase modulation with changing detuning frequency.
OIL-SRL to the phase of the injected light. It is due to the fact that the output phase of SRL tracks the changes in the phase of injected light from the master laser.

When the detuning frequency is increased from the negative edge to the positive of locking range, due to increase in the resonance frequency enhancement in the resonance peak is observed. But the resonance peak narrows because the damping factor decreases with increasing detuning frequency as shown in Fig. 3. Figure 3c shows the response as a linear function of ratio of output phase to input light phase.

\section{Conclusion}

Frequency response of master laser modulated OIL-SRL under direct, amplitude and phase modulation are derived and simulated. It is observed that the frequency response of OIL-SRL depends on detuning frequency and external optical injection ratio between the master laser and the slave SRL.

\section{Acknowledgments}

This research is supported by NPST program project No. 09-ELE857-02.

\section{References}

[1] E.K. Lau, L.J. Wong, M.C. Wu, IEEE J. Sel. Top. Quantum. Electron. 15, 618 (2009)

[2] M. Sorel, G. Giuliani, A. Scirè, R. Miglierina, S. Donati, P.J.R. Laybourn, IEEE J. Quantum Electron. 39, 1187 (2003)

[3] M.I. Memon, Bei Li, G. Mezosi, Z. Wang, M. Sorel, S. Yu, IEEE Photon. Technol. Lett. 21, 1792 (2009) 